\title{
Archéopages
}

Archéopages

Archéologie et société

$44 \mid 2017$

Terrains vagues

\section{Les « terrains vagues » de l'âge du Fer}

Le cas des oppida celtiques

The "vacant lots" of the Iron Age. The case of Celtic oppida

Los «Terrenos baldíos» de la Edad del Hierro. El caso de los oppida célticos

\section{Caroline von Nicolai}

\section{OpenEdition}

Journals

Édition électronique

URL : https://journals.openedition.org/archeopages/1464

DOI : 10.4000/archeopages. 1464

ISSN : 2269-9872

Éditeur

INRAP - Institut national de recherches archéologiques préventives

Édition imprimée

Date de publication : 1 juin 2017

Pagination : 6-15

ISSN : 1622-8545

Référence électronique

Caroline von Nicolai, « Les « terrains vagues » de l'âge du Fer », Archéopages [En ligne], 44 | 2017, mis en ligne le 01 juillet 2019, consulté le 02 juin 2021. URL : http://journals.openedition.org/archeopages/ 1464 ; DOI : https://doi.org/10.4000/archeopages. 1464 


\section{Les « terrains vagues » de l'âge du Fer \\ Le cas des oppida celtiques}

Caroline von Nicolai Université Ludwig-Maximilian de Munich, UMR 8546 «AOrOc»

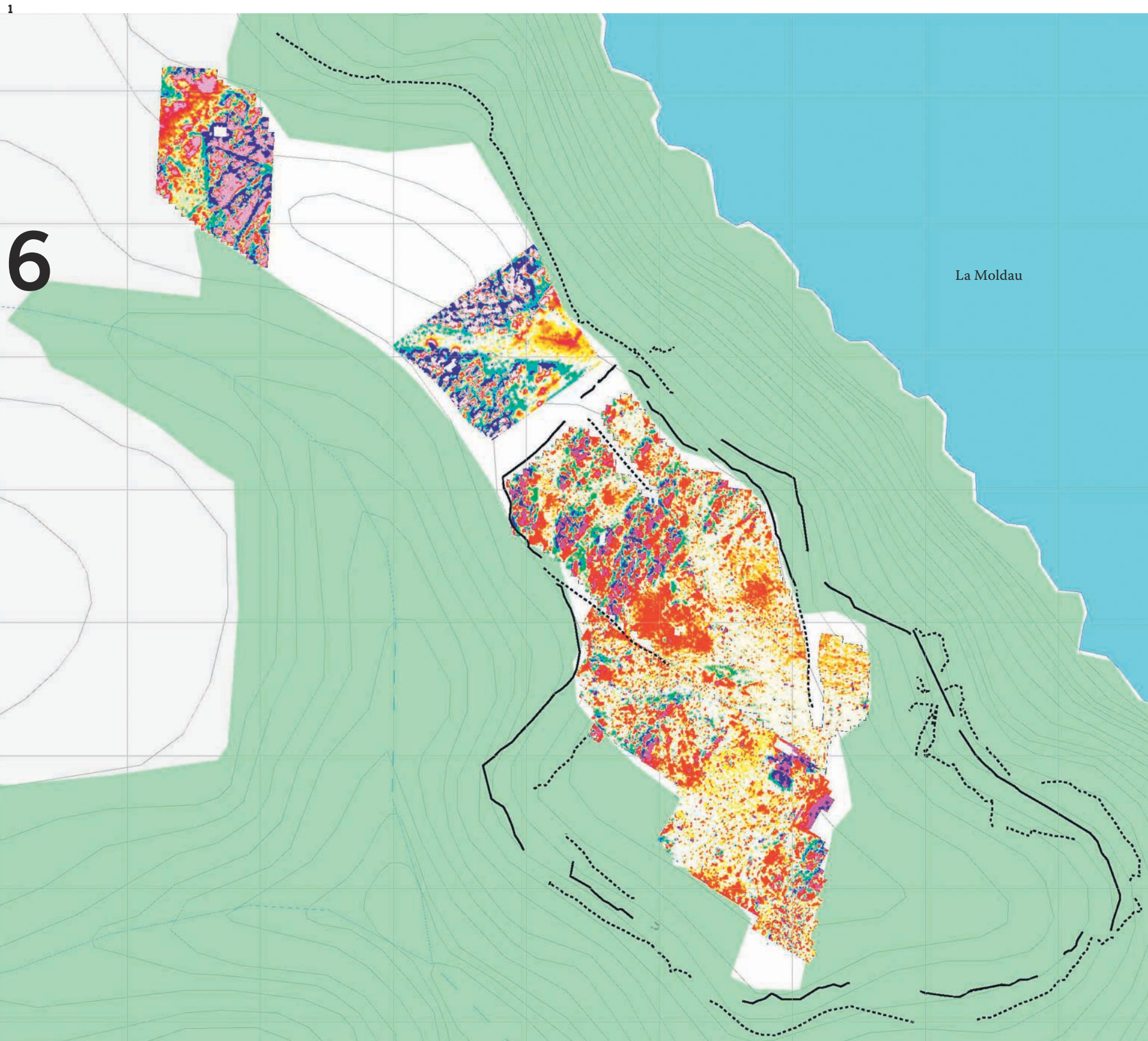


Les oppida celtiques : état de la recherche

Dès la deuxième moitié $\mathrm{du} \mathrm{II}^{\mathrm{e}}$ siècle avant notre ère se sont développées en Europe celtique de véritables villes, appelées habituellement oppida. Par ce terme, emprunté au De Bello Gallico de César, les protohistoriens désignent généralement des sites urbains et fortifiés des II $^{\mathrm{e}}$ et $\mathrm{I}^{\mathrm{er}}$ siècles avant notre ère en Europe tempérée, qui étaient situés pour la plupart en hauteur et caractérisés par la présence d'un rempart, d'un fossé et de portes souvent monumentaux (Gruel,

Buchsenschutz, 2015). À la différence des sites de hauteur fortifiés des époques antérieures, qui s'appuyaient généralement sur le relief et barraient des endroits défendus naturellement, les fortifications des oppida se libèrent des contraintes topographiques. Dans le but d'établir des lignes droites et continues et d'adopter des formes géométriques, elles dévalaient souvent les pentes perpendiculairement aux courbes naturelles du terrain. Leurs tracés pouvaient atteindre plusieurs kilomètres de long, entourant ainsi des surfaces qui s'échelonnaient de vingt-cinq à plusieurs centaines d'hectares (Fichtl, 2005). Certains oppida en particulier sortaient du lot en ce qui concerne leur superficie : l'enceinte de contour de Villejoubert dans le Limousin, par exemple, encerclait un éperon délimité par la confluence de la Vienne et de la Maulde. La surface ainsi enclose dépassait les 300 ha ; il s'agit donc du plus grand site de France datant de l'époque de La Tène (Ralston, 1992). Le site d'Altenburg-Rheinau (Bade-Wurtemberg en Allemagne et canton de Zurich en Suisse) se situait sur deux méandres du Rhin ; ses fortifications enserraient une superficie de 318 ha au total. L'oppidum de plaine de Manching en Bavière, dont l'enceinte décrit un cercle presque parfait, occupait 380 ha ; celui de Kelheim, également localisé en Bavière, environ 600 ha. L'oppidum le plus grand connu à ce jour est le « Heidengraben » (« le Fossé des Païens » en allemand) près de Grabenstetten au Bade-Wurtemberg dont les remparts barraient une surface d'environ 1770 ha (Stegmaier, 2014). En dehors de leur taille, une autre particularité caractérise beaucoup des oppida de La Tène finale : ce sont des fondations ex nihilo, ils sont construits à des endroits qui n'étaient pas occupés pendant la période précédente. On assiste même, dans certains cas, au déplacement d'un habitat ouvert de plaine au profit d'un habitat fortifié et situé en hauteur, comme c'est le cas du village des Arènes à Levroux (Indre), qui a été abandonné dans le deuxième quart du $\mathrm{I}^{\mathrm{er}}$ siècle avant notre ère en faveur de l'oppidum de la Colline des Tours, situé à proximité (Buchsenschutz et al., 2000). Dans d'autres cas, les oppida se sont apparemment développés à partir d'un lieu de culte plus ancien (Metzler et al., 2006).

Ces deux caractéristiques des oppida - la fondation ex nihilo et la superficie démesurée d'un certain nombre de sites - semblent, au premier coup d'œil, en faire des candidats particulièrement aptes pour aborder la notion des « terrains vagues » à l'âge du Fer. Dès l'époque de Joseph Déchelette (fin du XIX ${ }^{\mathrm{e}}$ et début du $\mathrm{XX}^{\mathrm{e}}$ siècle), auquel remonte la notion de la « civilisation des oppida », les chercheurs ont en effet postulé non seulement l'existence de quartiers d'habitat, d'artisanat et de commerce à l'intérieur des remparts monumentaux, mais encore de zones « vides », dépourvues de toute occupation. Ces aires étaient censées servir tantôt, en période de paix, comme zones de pâturage pour les bestiaux, tantôt, en période de crise, pour accueillir la population des campagnes environnantes (Déchelette, 1914). Vu l'état de la recherche, il est pourtant difficile de faire des constatations aussi générales sur l'utilisation des terrains enclos par les fortifications, car, en raison de leur taille, aucun oppidum n'a fait l'objet d'une fouille exhaustive jusqu'à nos jours. Dans l'oppidum géant de Heidengraben, par exemple, moins d'1 \% des 1770 ha enclos ont été fouillés jusqu'en 2014; le reste n'a été exploré que par les prospections pédestres et géophysiques (Stegmaier, 2014). Même les oppida les mieux étudiés, tels que Manching en Bavière ou Bibracte en Bourgogne, n'ont été fouillés que très partiellement (3o ha, soit $7 \%$ pour Manching ; 6 \% pour Bibracte en 2012). Tenant compte du budget financier et de la dépense de temps nécessaires à une telle entreprise, il est peu probable qu'un oppidum soit jamais fouillé intégralement dans l'avenir (Fichtl, 2012). Même les prospections géophysiques et les images LIDAR (Light Detection and Ranging), qui peuvent également révéler des traces d'occupation et sont plus rapides et moins coûteuses que les fouilles, comprennent rarement la totalité des sites en question. Une des seules exceptions est l'oppidum du Titelberg au Luxembourg, où une prospection géomagnétique, une couverture LIDAR et des photographies aériennes ont permis de faire ressortir la grille urbaine du site, par exemple les rues, les fossés de délimitation intérieurs, ainsi qu'un certain nombre de bâtiments (Metzler, 2008). En République tchèque également, l'Institut d'archéologie de l'Académie des sciences a effectué des prospections géophysiques de grande envergure, entre 2003 et 2007, dans cinq oppida (Hrazany, Nevězize, Stradonice, Tř́ísov et Závist), portant sur des surfaces entre 5 et 23 ha par site, au total 67 ha. À Nevězize, par exemple, la moitié de la surface de l'oppidum (7,45 ha de la surface totale de 13 ha), qui se trouve sur un éperon barré surplombant la Vltava, a été étudiée en appliquant différentes méthodes, à savoir la prospection géomagnétique, la prospection par résistivité électrique et la prospection par conductivité du sol, en combinaison avec l'étude des photographies aériennes et des prospections pédestres, avec et sans détecteurs à métaux. Les résultats sont prometteurs car ces prospections ont permis de faire ressortir des tracés de fortifications et des

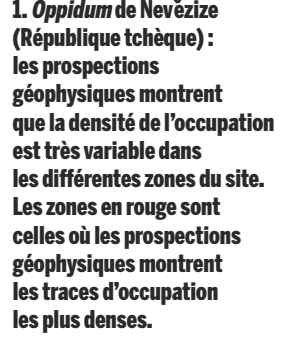


Une multitude de bâtiments

(en valeurs de rouge

différentes selon la

chronologie) de taille

et de forme diverses

sont présents dans

cette partie de l'oppidum.

Ils s'alignent le long

des axes de circulation.
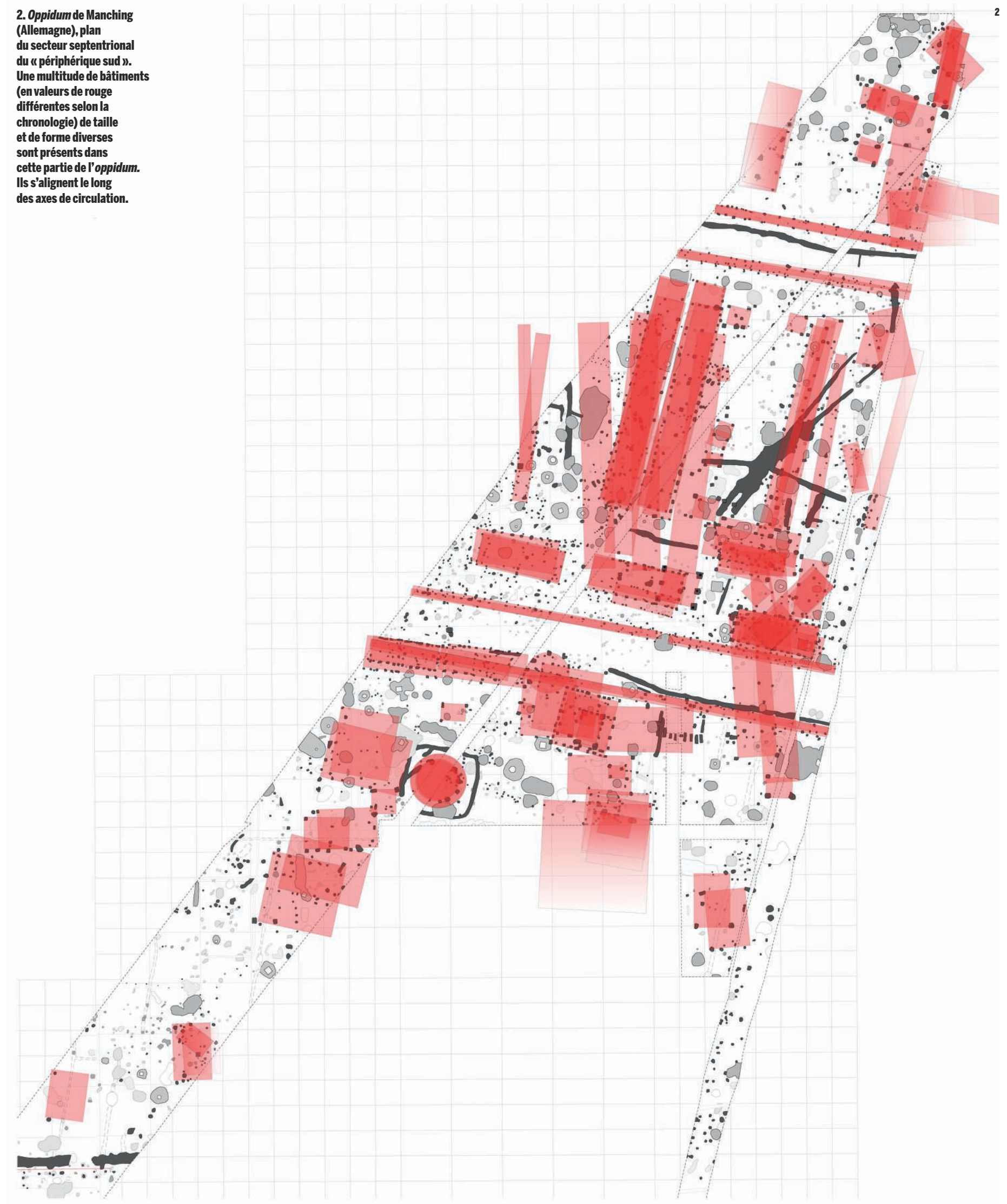
portes, des structures linéaires, qui correspondent probablement à des enclos fossoyés, des axes de circulation, des plateformes et des terrasses artificielles, des fosses, des bâtiments et des installations artisanales (fours, accumulation de déchets de production, etc.), mais aussi des secteurs apparemment « vides » [ill. 1]. En s'appuyant seulement sur les données issues des prospections géophysiques, sans fouiller les zones concernées, il est pourtant impossible de déterminer si celles-ci étaient intentionnellement laissées libres dans l'Antiquité, si les surfaces étaient trop érodées pour révéler encore des traces d'occupation ou si les méthodes appliquées étaient inadéquates, par exemple à cause de la géologie ou des sols locaux (Křivánek et al., 2013). Malgré ces avancées de la recherche, l'organisation interne de la plupart des oppida reste par conséquent en grande partie inconnue. On ignore donc souvent si des zones, que nous qualifions de « vagues » à l'heure actuelle, l'étaient vraiment pendant l'âge du Fer ou si cette caractérisation reflète seulement l'état de nos connaissances. C'est à partir de cette question que cet article, qui essaye de résumer l'état de la recherche concernant la question des «terrains vagues » dans les oppida celtiques de la fin de l'âge du Fer, doit être lu.

\section{L'organisation interne des oppida}

Pour comprendre la fonction des « zones vides » dans les oppida, il semble utile d'examiner plus en détail, dans la mesure du possible, les plans et l'organisation internes des sites, tels qu'ils sont révélés par les fouilles et les prospections. La plupart des sites possédaient, semble-t-il, des axes de circulation principaux et montraient une subdivision en plusieurs zones. À Bibracte dans le Morvan, par exemple, une voie principale, dite « Rue du Rebout », reliait les portes principales de l'oppidum. Des bâtiments, souvent arrangés en îlots, étaient alignés le long de cette voie. En revanche, l'idée, formulée au début du $\mathrm{XX}^{\mathrm{e}}$ siècle par Joseph Déchelette (Déchelette, 1914), que le site était clairement divisé en quartiers, dont certains étaient des secteurs résidentiels, tandis que d'autres étaient dédiés uniquement à l'artisanat, peut aujourd'hui être rejetée. Il semble plutôt que de petits ateliers étaient dispersés dans toute la ville, parmi les maisons d'habitation, car l'analyse des distributions des outils et des déchets de production en fer n'a fait ressortir ni des quartiers spécialisés, ni des concentrations par métier artisanal (Mölders, 2009). À VilleneuveSaint-Germain dans l'Aisne, oppidum de 70 ha localisé sur une terrasse fluviatile dans un méandre de l'Aisne, deux fossés rectilignes et perpendiculaires, se croisant dans l'angle sud-est du site, subdivisaient l'ensemble de l'habitat en quatre parties inégales. Ces fossés, longs de plusieurs centaines de mètres, étaient probablement recouverts d'une toiture, formant ainsi une sorte de galerie de déambulation. Entre ces grands axes étaient situés des enclos fossoyés ou îlots, enserrant chacun un bâtiment d'habitation, ses annexes et des fosses à caractère domestique ou artisanal. L'occupation la plus dense de La Tène finale semble se concentrer sur une terrasse non inondable de l'Aisne, d'une vingtaine d'hectares. En revanche, la plaine alluviale d'une cinquantaine d'hectares au fond de la presqu'île, qui était submergée lors des fortes crues, a livré beaucoup moins de mobilier (mais ce secteur n'a pas encore été fouillé, à vrai dire) (Debord et al., 1988). À Manching, des zones densément occupées, situées notamment au centre de l'oppidum, où les bâtiments étaient proches les uns des autres, alternaient avec des secteurs beaucoup moins aménagés où des installations de type ferme prédominaient. Celles-ci étaient séparées l'une de l'autre par des fossés, des clôtures et des chemins, rappelant ainsi l'occupation des campagnes (Sievers, 2010). Même dans un petit secteur, comme celui du « périphérique sud» de Manching, fouillé entre 1965 et 1971, les types de construction étaient très variés : des bâtiments à deux nefs, longs de $43 \mathrm{~m}$ et larges de $6 \mathrm{~m}$, étaient érigés à proximité de bâtiments rectangulaires à deux pièces, de $16 \mathrm{~m}$ sur $6 \mathrm{~m}$, mais aussi de greniers à quatre ou à six poteaux, de bâtiments circulaires et de bâtiments semi-enterrés [ill. 2] (Winger, 2015). Une agglomération d'unités domestiques individuelles à caractère rural est également connue dans l'oppidum de Staré Hradisko en République tchèque [ill. 3]. La fouille extensive de cet établissement rural a montré que l'agglomération était composée de plusieurs bâtiments à poteaux, de bâtiments semi-enterrés, de greniers, de citernes, de fours de potier et de foyers. Enserré dans un enclos, cet établissement ne se distinguait donc pas d'une ferme localisée en pleine campagne (Danielisová, Hajnalová, 2014). Ces quelques exemples montrent que la plupart des oppida présentaient des trames urbaines plutôt lâches, avec un habitat clairsemé, où les terrains non construits étaient nombreux. À quoi servaient-ils?

\section{Les places publiques à l'intérieur des oppida}

Certains terrains vagues situés à l'intérieur des remparts urbains fonctionnaient, selon toute vraisemblance, comme places ouvertes. Un des meilleurs exemples connus est l'oppidum du Titelberg, qui appartenait à la cité des Trévires et en était probablement la capitale [ill. 4a]. Cet oppidum de 43 ha était implanté sur un éperon dominant la vallée de la Chiers et entouré d'un murus gallicus, érigé vers 100 avant notre ère. L'occupation était assez dense dans la partie occidentale du site. Dans la partie orientale, en revanche, un espace était séparé du reste de l'habitat par un mur en briques crues, ainsi que par un fossé d'une longueur de $500 \mathrm{~m}$, d'une largeur de 4. m et d'une profondeur de 2,5 m, creusé dans le rocher vers 100 avant notre ère. La plus 

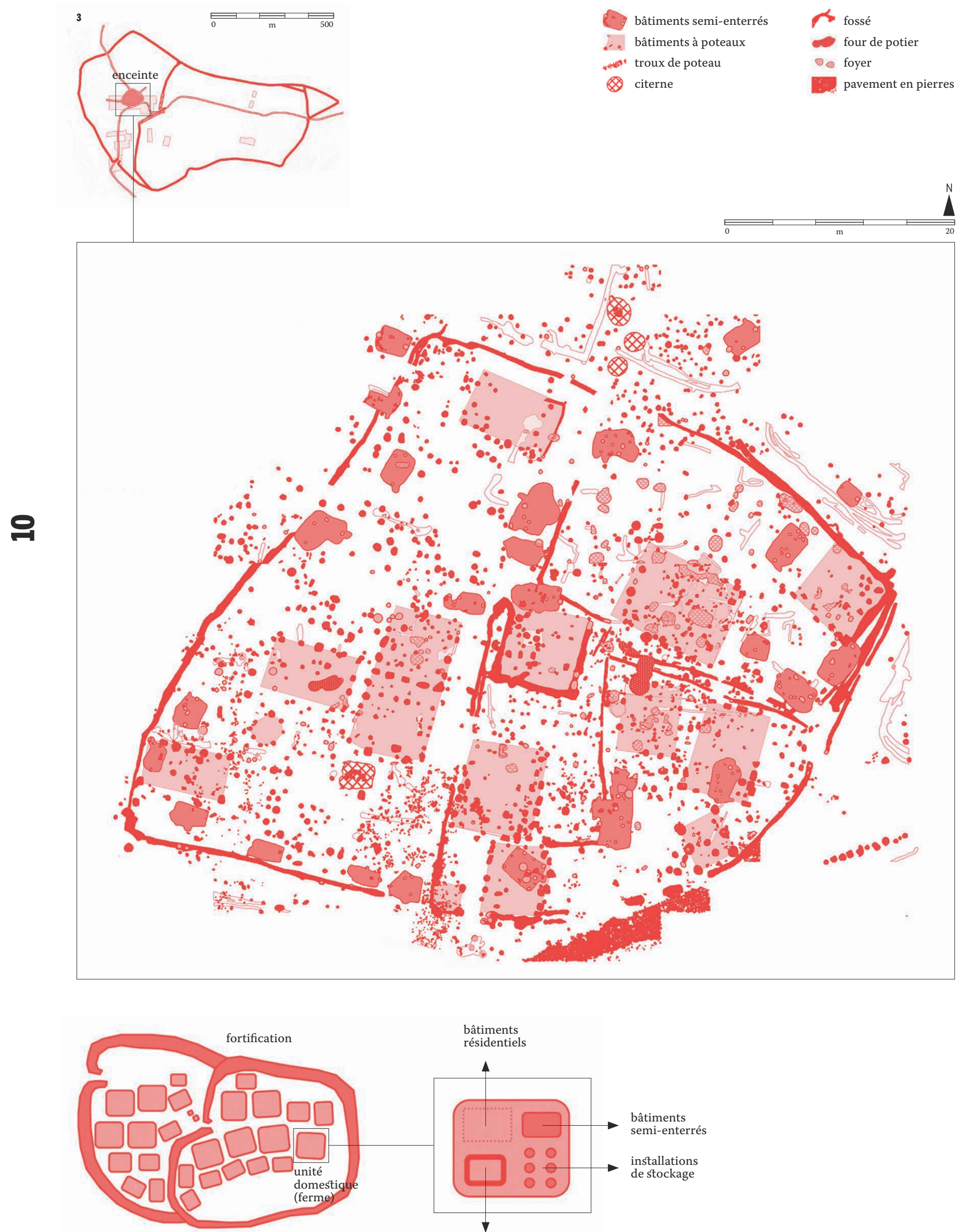

Oppidum

bâtiments

résidentiels

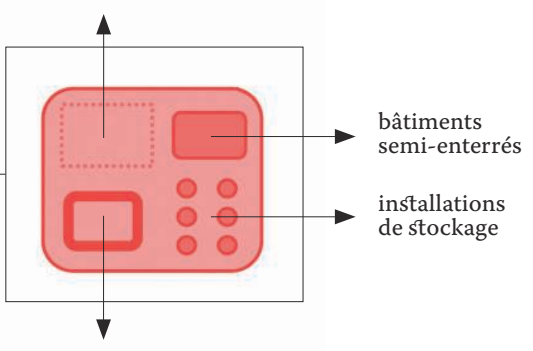

ateliers

artisanaux 
grande partie de cette enceinte n'était pas construite ou aménagée ; la partie la plus élevée de l'oppidum, dans l'axe qui passait entre les deux portes de l'oppidum, présentait pendant la première moitié $\mathrm{du} \mathrm{I}^{\mathrm{er}}$ siècle avant notre ère une série de palissades parallèles, qui formaient des couloirs larges d'environ $4 \mathrm{~m}$ et longs d'au moins $60 \mathrm{~m}$, orientés perpendiculairement à la voie principale de l'oppidum. Ces palissades ont été démolies et reconstruites plusieurs fois durant l'occupation du site. Dans le deuxième quart du $\mathrm{I}^{\text {er }}$ siècle avant notre ère, elles étaient remplacées par un bâtiment ouvert à trois nefs, de $14 \mathrm{~m}$ sur $15 \mathrm{~m}$, précédé d'une esplanade vide de $40 \mathrm{~m}$ de long, bordée elle-même d'un bâtiment ouvert à nef unique [ill. 4b]. Devant cette grande halle se trouvait un parvis, une fondation carrée en plaques de calcaire (un autel ?) et des foyers. Pendant les années 20 avant notre ère, probablement lors de la fondation de la nouvelle capitale des Trévires, Augusta Treverorum, à proximité de l'oppidum, ce bâtiment a été démoli et le fossé entourant le site a été comblé pour y aménager un pavage avec plusieurs foyers. Dans le courant du deuxième quart du $\mathrm{I}^{\text {er }}$ siècle de notre ère, un deuxième bâtiment ouvert en pierres fut érigé au même emplacement, avant d'être lui-même remplacé par un large fanum, un temple gallo-romain maçonné, au II $^{\mathrm{e}}$ siècle. En raison de cette succession de constructions dans la partie la plus élevée de l'oppidum, ainsi que des vestiges particuliers retrouvés à cet endroit, dont environ 100 ooo ossements animaux, une centaine de fibules, des pointes de lance, quelques armes miniaturisées en bronze, une série de rouelles et de nombreuses céramiques, Jeannot Metzler et ses collègues, qui ont fouillé le site, interprètent ce vaste terrain vague comme un large centre polyvalent, séparé de l'habitat par une enceinte. Cette aire abritait, d'après les résultats des prospections géomagnétiques et des sondages, non seulement une grande halle, remplacée plus tard par un temple, mais aussi un grand espace qui ne fut jamais aménagé. Cet espace polygonal était probablement utilisé pour les réunions publiques, politiques et cultuelles de la cité des Trévires, telles que César les décrit dans la Guerre des Gaules (De Bello Gallico V, 56) mais aussi pour la tenue de grands marchés et de foires (Metzler, 2006 ; Metzler et al., 2006 ; Metzler, 2008). Les palissades décrites ci-dessus ont été interprétées comme des couloirs de vote, à l'exemple des saepta des villes italiques, connues par exemple à Rome, Fregellae ou Paestum. Ces structures amovibles étaient installées pour canaliser les électeurs pendant les assemblées publiques (Metzler, 2006 ; Fichtl, 2010 ; Fernández-Götz, 2014a ; Fernández-Götz, 2014b).

Des places où des réunions politiques, des activités cultuelles mais aussi des marchés pouvaient se dérouler sont aussi attestées dans d'autres oppida, comme celui du Martberg, également situé en pays trévire dans la vallée de la Moselle. Les fouilles ont mis en évidence un espace non construit de $50 \mathrm{~m}$ sur $60 \mathrm{~m}$ au sommet de l'oppidum, qui était entouré d'une palissade. $\mathrm{Au}$ centre se trouvait un enclos de $10 \mathrm{~m}$ sur $12 \mathrm{~m}$, délimité par un fossé. Le mobilier découvert (des milliers de monnaies et des centaines de fibules) a permis d'établir que cette place à vocation probablement publique a été aménagée pendant la première moitié du $\mathrm{I}^{\mathrm{er}}$ siècle avant notre ère, pendant La Tène D1. Plus tard, à la fin du $\mathrm{I}^{\mathrm{er}}$ siècle et au début du $\mathrm{II}^{\mathrm{e}}$ siècle de notre ère, les habitants du Martberg y érigeaient les premiers temples en pierres, monumentalisant ainsi l'ancien centre politique et religieux du site (Nickel et al., 2008). À Corent en Auvergne, la place centrale, appelée « esplanade » par les fouilleurs, atteignait une surface de $70 \mathrm{~m}$ de longueur et $60 \mathrm{~m}$ de largeur, c'est-à-dire d'environ $4,000 \mathrm{~m}^{2}$. La surface de cette esplanade correspondait au niveau de la roche basaltique naturelle, terrassé et laissé à nu, ce qui lui conférait l'aspect d'un dallage artificiel en pierres. La place était bordée à l'ouest, dès la seconde moitié du $\mathrm{II}^{\mathrm{e}}$ siècle avant notre ère, d'un sanctuaire monumental, et sur les trois autres côtés par des bâtiments alignés. Ceux-ci formaient des îlots d'environ $30 \mathrm{~m}$ par $20 \mathrm{~m}$ de côté, bâtis densément, séparés l'un de l'autre par des ruelles. Le mobilier archéologique découvert permet de constater que ces îlots étaient surtout destinés à l'habitat et à l'artisanat. Au sud-est du sanctuaire, les fouilles ont mis au jour une construction en bois en hémicycle, à laquelle a été substituée à l'époque romaine une construction similaire en pierres. La construction ressemble à celle d'un petit théâtre romain ou d'un comitium, c'est-à-dire un lieu de réunion civique (Poux, Demierre, 2015). Souvent associées à des sanctuaires, que l'on transformait pour la plupart à l'époque galloromaine en temples, les places publiques des oppida semblent donc avoir joué un rôle prépondérant pour rassembler des foules nombreuses lors des cérémonies publiques et religieuses, des réunions politiques, des foires et des marchés. Ainsi, elles permettaient de constituer et de maintenir des relations sociales et collectives au sein des cités gauloises, forgeant de cette façon une identité communautaire propre à chaque communauté (Fernández-Götz, 2012 ; Fernández-Götz, 2014b). Ces places pouvaient rester sans aménagement, et par conséquent des « terrains vagues », jusqu'à l'abandon définitif des habitats en question.

\section{L'agriculture au sein des habitats}

Une partie des « terrains vagues » situés à l'intérieur des fortifications semblent avoir été utilisés à des fins très différentes, à savoir pour l'agriculture et l'élevage. C'est le cas de Manching où non seulement les fouilles mais aussi les études archéobotaniques ont révélé la présence de champs à l'intérieur de l'oppidum. Certains secteurs au centre du site, fouillés entre 1984 


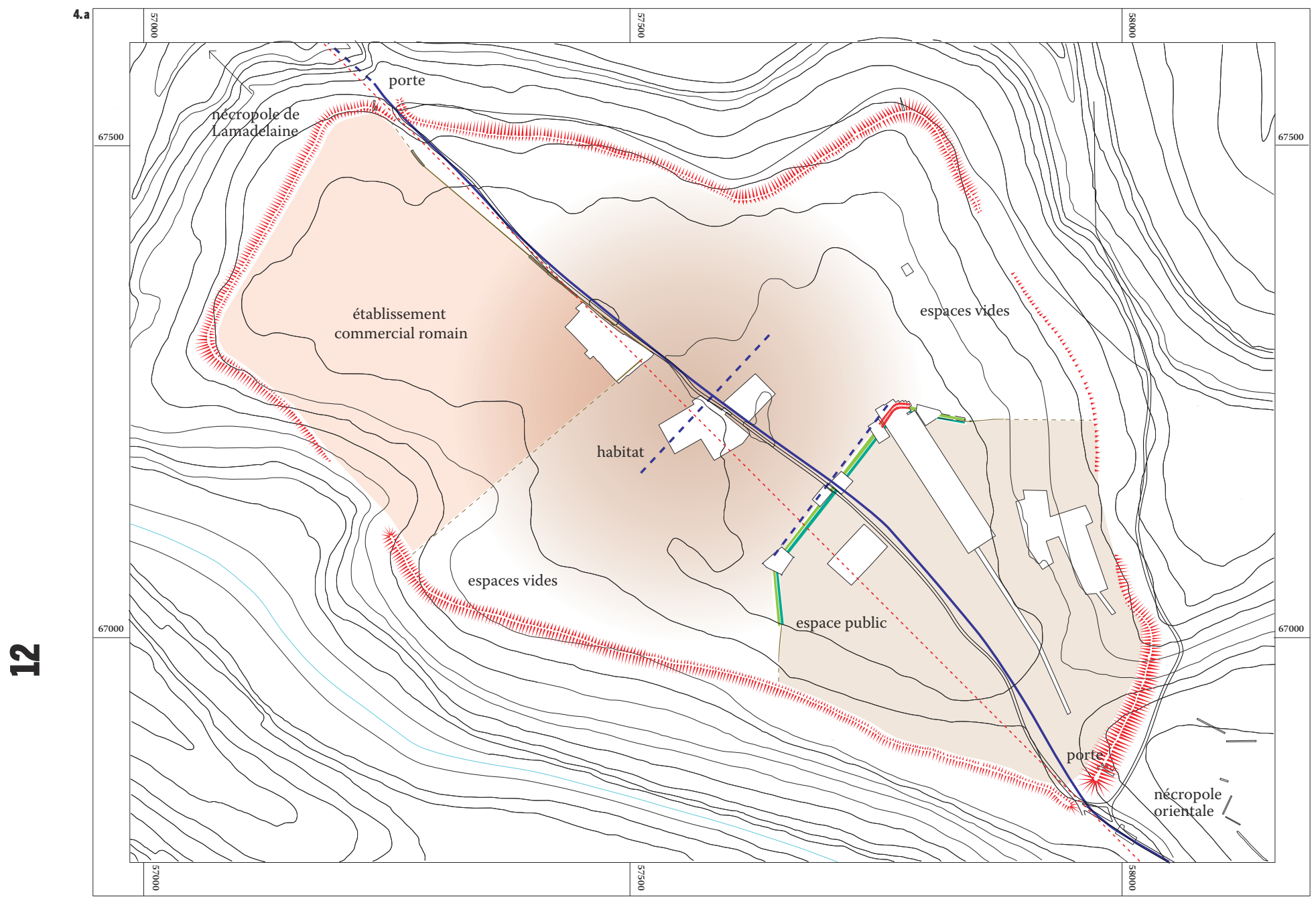

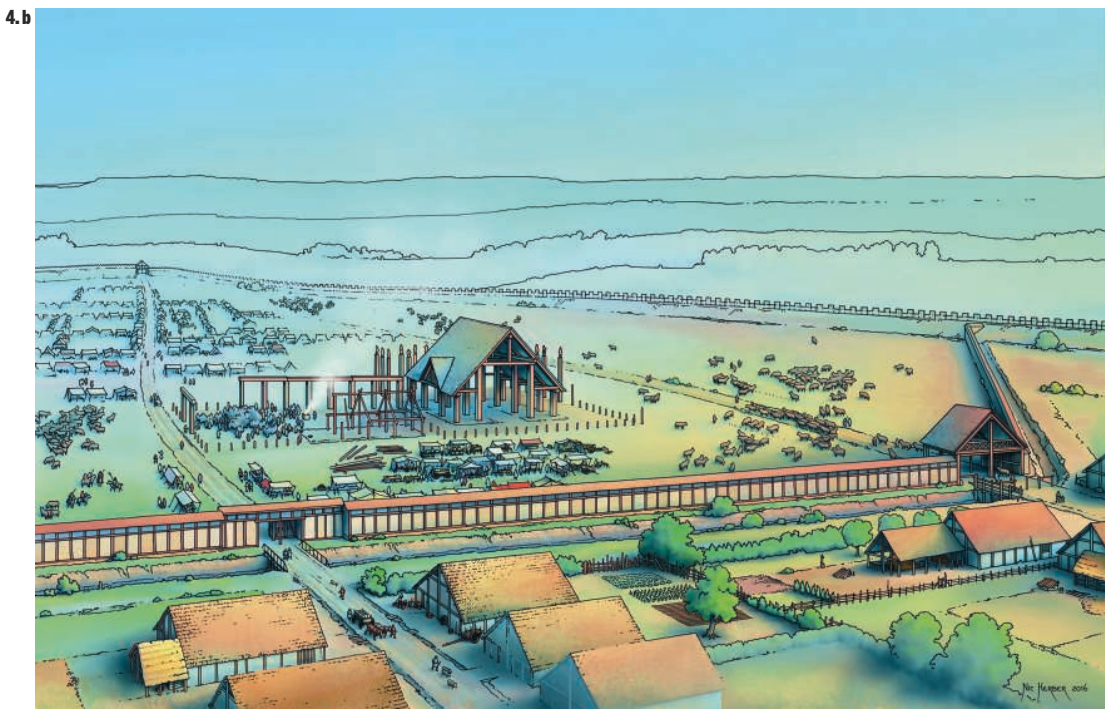

4. a. Oppidumdu Titelberg (Luxembourg). Le site comprend deux zones

séparées l'une de l'autre par un mur (en vert):

l'habitat et un espace public.

Aux marges de l'habitat

se trouvent des espaces

vides, qui ont livré très peu

de vestiges archéologiques.

Ils ont peut-être été utilisés

comme pâturages, jardins

potagers ou vergers.

Les espaces blancs sont

les zones fouillées.

4. b. L'espace public de

l'oppidum du Titelberg

(par rapport au plan 4. a, vue

du nord-ouest vers le sud-

est), utilisé probablement

pour les réunions publiques,

politiques et cultuelles de la

cité des Trévires, est dominé

par une grande halle dans

le deuxième quart du ${ }^{\text {er siècle. }}$
5. Oppidum de Heidengraben (Allemagne). L'habitat (E, en rose), entouré de tous les côtés de fortifications (en rouge) se concentre dans I' "I Elsachstadt " ".

À l'extérieur de celle-ci,

des sols très fertiles (en vert) sont présents dans certaines parties du site. Sur ceux-ci,

des concentrations de

mobilier (dont des amphores et des céramiques) ont été rencontrées; elles signalent

l'existence de fermes. 


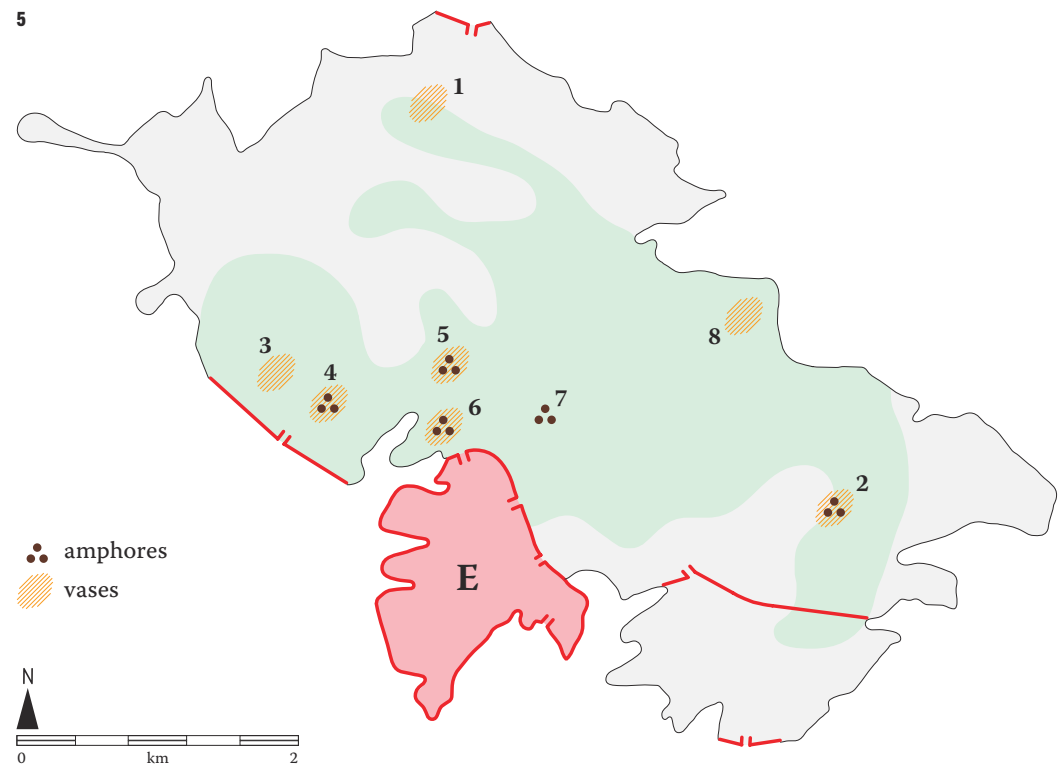

et 1987, sont caractérisés par des réseaux de petits fossés encadrant des surfaces plus ou moins rectangulaires de plusieurs ares chacune. Ces fossés servaient probablement au drainage du terrain. Le sol délimité par ces fossés était souvent en forme de voûte, signalant des labours ${ }^{\mathbf{1}}$. En outre, l'analyse des échantillons de sédiments provenant de ces secteurs a montré que ceux-ci étaient riches en plantes adventices, tandis que ceux provenant des zones occupées par des bâtiments contenaient surtout des grains de blé et des glumes. Cette différence pourrait indiquer que les habitants de l'oppidum cultivaient, séchaient et décortiquaient des céréales dans certaines parties du site, probablement des champs, tandis qu'ils stockaient les grains dans d'autres, à proximité de leurs maisons d'habitation. Si l'on considère le fait que l'oppidum était entouré en grande partie par des tourbières basses et par des cours d'eau et que la plupart des terres cultivables étaient situées à l'intérieur du rempart, la présence de champs près des quartiers d'habitat est d'autant plus probable (Küster, 1992).

Les fouilles et les analyses archéobotaniques entreprises à Manching entre 1996 et 1999 dans le secteur dit Altenfeld confirment cette hypothèse : à l'intérieur de l'enceinte, il existait et des quartiers d'habitat, de commerce et d'artisanat densément peuplés, et des fermes et des champs où l'on cultivait de l'orge, de l'amidonnier, du blé, de l'épeautre, des haricots, des lentilles et de l'oeillette (Küster, 2013). Il en va de même pour la zone dite «périphérique sud » de l'oppidum de Manching, où les fouilleurs ont identifié non seulement l'existence de champs en marge des quartiers d'habitat, près du rempart, mais encore l'élevage de bœufs à l'intérieur du rempart, dans un grand enclos qui s'est révélé particulièrement riche en ossements animaux (Winger, 2015). La situation dans l'oppidum de Heidengraben est comparable. Occupant une surface de 1770 ha, il s'agit de loin de l'oppidum le plus grand connu à ce jour. Néanmoins, les fouilles partielles et les récoltes de surface indiquent que seul un secteur du site dit l'«Elsachstadt», qui couvre une surface de 165 ha et est séparé du reste du site par une deuxième fortification, semble avoir été occupé de manière dense dès la deuxième moitié du $\mathrm{II}^{\mathrm{e}}$ siècle avant notre ère (Stegmaier, 2014). À l'extérieur de ce noyau « urbain », le mobilier archéologique est beaucoup moins abondant, quoique certaines concentrations d'objets (céramique tournée et non tournée, objets métalliques, fragments d'amphores, etc.) pourraient indiquer la présence de fermes isolées [ill. 5]. Celles-ci s'échelonnaient sur 800 ha de bonnes terres labourables et très fertiles selon les standards de l'agronomie moderne, situées à l'intérieur de l'enceinte extérieure (Stegmaier, 2014). En outre, un nombre élevé d'ossements de bœuf retrouvés lors des fouilles pourrait témoigner non seulement de l'importance du cheptel bovin au Heidengraben, mais encore de l'existence de pâturages à l'intérieur des fortifications du site (Uerpmann, Uerpmann, 2006). Une interprétation semblable est proposée pour la partie occidentale (dite «Westwerk») de l'oppidum du Donnersberg en Rhénanie-Palatinat dont la surface totale s'élève à 240 ha. Le terrain irrégulier du « Westwerk », marqué par des ravines profondes, se prêtait en effet peu à l'occupation et le mobilier archéologique récolté en surface y est peu abondant. C'est probablement aussi pour cette raison que les fortifications y étaient moins puissantes et n'étaient pas restaurées, à la différence des remparts de l' «Ostwerk » (le secteur oriental de l'oppidum) où se sont concentrées les découvertes archéologiques et où était donc situé selon toute vraisemblance aussi l'habitat. À la différence de Manching ou du Heidengraben, l'hypothèse d'une utilisation agricole d'une partie du territoire n'est pourtant pas confirmée par les études archéobotaniques pour le Donnersberg à l'heure actuelle (Zeeb-Lanz, 2016).

\section{L'extraction de matières premières à l'intérieur des fortifications}

Les fortifications des oppida pouvaient aussi enserrer des sites d'extraction de matières premières, comme l'oppidum de Kelheim en Bavière le montre. Situés à la confluence du Danube et de l'Altmühl, sur un éperon dit « Michelsberg » ou « Hirschberg », ses murs protégeaient une surface de plus de 600 ha. Loccupation se concentrait, selon toute vraisemblance, derrière la fortification interne dans la partie orientale de l'oppidum, dite « Mitterfeld». Entre la fortification interne et la fortification extérieure s'étendait une vaste zone sans traces d'occupation où de nombreuses fosses attestent l'extraction du minerai de fer pendant l'époque celtique. Les riches gisements de minerai s'y trouvaient très proches de la surface et étaient donc faciles à exploiter à ciel ouvert, en creusant 
des fosses plus ou moins circulaires, larges de $15 \mathrm{~m}$ et profondes de $7 \mathrm{~m}$ au maximum. Environ 2000 de ces fosses datent probablement de l'époque laténienne ; le reste peut être attribué au Moyen Âge. Deux fourneaux pour la transformation du minerai de fer, découverts dans le Mitterfeld, devant le mur externe du site, des crassiers, des scories, des outils pour le travail du fer, des lingots de fer ainsi que des déchets de production témoignent également de l'importance des activités sidérurgiques dans l'oppidum (Schwarz et al., 1965-1966 ; Rind, 1994; Tuschwitz, 2015). Dans l'état actuel de la recherche, l'oppidum de Kelheim paraît pourtant sans pareil dans le monde celtique. Une exploitation du minerai de fer et des activités sidérurgiques ont certes également été présumés pour l'oppidum de Heidengraben (voir ci-dessus), au vu des dimensions du site (par exemple Fischer, 2001). Pourtant, cette hypothèse ne peut plus être retenue si l'on tient compte des résultats des recherches les plus récentes sur la production du fer dans le sud-ouest de l'Allemagne à l'âge du Fer (Stegmaier, 2014).

\section{Des fortifications dépourvues de toute occupation?}

Outre les fortifications surdimensionnées, qui renfermaient, en plus des habitations, des lieux publics et des ateliers, ainsi que des champs ou des sites d'extraction de matières premières, il existe aussi des sites dont la fonction reste vague, parce que les découvertes archéologiques y sont très rares. À Kirchzarten, à l'est de Fribourg-en-Brisgau au Bade-Wurtemberg, par exemple, se trouve une vaste fortification de La Tène finale (également appelée « Heidengraben »), qui est souvent identifiée comme le Tarodunum mentionné par le géographe grec Ptolémée au $\mathrm{II}^{\mathrm{e}}$ siècle de notre ère. Son murus gallicus encercle un éperon barré de 190 ha. À l'intérieur du rempart, les traces d'occupation font quasiment défaut, bien que le site ait fait l'objet de plusieurs fouilles. Celles-ci ont montré que la construction du murus gallicus n'a jamais été terminée, interrompue assez brusquement vers 100 avant notre ère pour des raisons qui restent inconnues (Dehn, 2005). Une agglomération non fortifiée légèrement plus ancienne, datant de La Tène moyenne et finale (Lt $C_{2}$ et $D_{1}$ ), a été repérée 800 m à l'ouest de la fortification, au lieu-dit « Rotacker ». Elle s'étendait sur une surface de 12 ha et a livré non seulement des restes de bâtiments, des fosses, une place pavée et un atelier de potier, mais aussi un grand nombre de monnaies, d'amphores d'origine romaine, de fragments de bracelets et de perles en verre, ainsi que des déchets d'activités métallurgiques qui attestent une production artisanale riche et variée et des relations commerciales à longue distance (Wendling, 2005 ; Wendling, 2006). L'espace enclos par la fortification voisine de Tarodunum constitue donc un terrain vague par excellence. Il correspond probablement à un projet avorté de création d'un oppidum car l'habitat n'a jamais été transféré à l'intérieur de la fortification.

Au Mont Vully dans le canton de Vaud, oppidum situé sur un promontoire naturel entre les lacs de Neuchâtel et de Morat en Suisse, la situation est comparable à celle de Tarodunum. Les fouilles entreprises entre 1978 et 2003 n'ont permis de mettre en évidence ni habitat pérenne, dense et structuré, ni voies intérieures, ni centre religieux, ni mobilier archéologique abondant à l'intérieur de la fortification qui enserrait une surface d'environ 50 ha. Cependant, à la différence de Tarodunum, le système défensif du Mont Vully était non seulement achevé mais encore très élaboré, comportant deux portes flanquées de tours. Cela porte les fouilleurs à croire que le site ne constituait pas une ville mais servait de forteresse, de refuge ou de lieu de réunion pour les habitants du territoire environnant ou d'une région plus étendue (Kaenel et al., 2004).

Ces quelques exemples montrent bien que ces zones vides situées à l'intérieur des fortifications étaient assez fréquentes dans les oppida celtiques des $\mathrm{II}^{\mathrm{e}}$ et $\mathrm{I}^{\mathrm{er}}$ siècles avant notre ère. Cela s'explique probablement par le fait que les oppida étaient souvent très grands, notamment par rapport aux sites fortifiés des époques précédentes, mais aussi par rapport à ceux des périodes suivantes. Les fonctions de ces terrains non aménagés et non construits à l'intérieur des remparts étaient en tout cas très variables. Certains peuvent être qualifiés de places publiques et de zones de passage, situés plutôt au centre des lieux d'habitat ou dans les parties les plus élevées des surfaces encloses. Quelquefois, des délimitations - des murs ou des palissades - sont attestées qui séparaient ces places du reste de l'habitat. En tant que lieux de rassemblement pour des activités politiques, religieuses et économiques, les places laissées délibérément libres étaient très importantes pour la vie communautaire des habitants. Il est par conséquent fort probable qu'une autorité a commandé leur aménagement lors de la création de l'habitat, selon un plan qui traduit la volonté de ses fondateurs d'organiser l'habitat rationnellement (Smith, 2008). D'autres espaces non construits - souvent localisés en marge des zones construites, proches des remparts - étaient probablement des champs, des pâturages ou des sites d'extraction de matières premières. Parfois, la nature du sol peut en effet expliquer l'emplacement des terrains vagues à l'intérieur des lieux d'habitat. Il est évident que les champs et les établissements ruraux se concentraient sur les sols fertiles, tandis que des aires humides et exposées aux inondations étaient plutôt utilisées comme pâturages. Certaines fortifications semblent même avoir enserré uniquement des terrains vagues, sans qu'une vraie occupation y soit attestée. Deux explications peuvent être proposées dans ces cas-là : soit il s'agit de projets d'urbanisation 
non aboutis, soit ces enceintes étaient avant tout des lieux de rassemblement, utilisés à des fins politiques ou comme refuges dans des situations difficiles. Malheureusement, l'état de la recherche et le défaut de sources textuelles pour l'âge du Fer ne permettent généralement pas de déterminer si ces aires vides étaient destinées à le rester ou si elles étaient réservées à de futurs programmes d'urbanisation. Dans ce dernier cas, l'aire enserrée par la fortification aurait été prévue, au moment de la fondation de l'oppidum, pour contenir une certaine population. La population s'avérant moins nombreuse qu'escompté, ces zones seraient restées vides. Parfois même, le site fortifié n'est pas occupé du tout, comme à Tarodunum. Contrairement aux places publiques, aménagées selon un plan préconçu, ces vastes zones restaient ainsi libres involontairement.

L'expérience urbaine des oppida est un phénomène très éphémère qui a duré à peine plus d'un siècle et demi, parfois encore moins. La conquête romaine mit fin au développement des oppida dans l'ouest du monde celtique, avec l'essor de villes à la romaine, dont l'urbanisme est bien différent de celui des villes celtiques. Dans l'est, les oppida étaient abandonnés dans le courant du $\mathrm{I}^{\mathrm{er}}$ siècle avant notre ère pour des raisons encore inconnues. Il est donc impossible de savoir comment ces terrains vagues à l'intérieur des oppida auraient été restructurés et remodelés dans la longue durée.
Références bibliographiques

César, De Bello gallico, Paris, Presses universitaires de France, 1974.

BuCHSENSCHutz O. et al., 200o, Le village celtique des Arènes à Levroux: synthèses, Levroux 5 , Tours, FERAC-ADEL, Supplément à la Revue archéologique $d u$ Centre de la France, 19, $333 \mathrm{p}$.

Danielisová A., Hajnalová M., 2014, « Oppida and agricultural production : state of the art and prospects. Case study from Staré Hradisko oppidum (Czech Republic) », in Hornung S. (dir.), Produktion - Distribution - Ökonomie. Siedlungs- und Wirtschaftsmuster der Latènezeit, Akten des internationalen Kolloquiums in Otzenhausen, 28.-30. Oktober 2011, Universitätsforschungen zur prähistorischen Archäologie, 258, Bonn, Habelt, p. 407-428.

Debord J., LAMBot B., Buchsenschutz O., 1988 «Les fossés couverts du site gaulois tardif de Villeneuve-Saint-Germain (Aisne) », in Audouze F., Buchsenschutz O. (dir.), Architectures des âges des métaux: fouilles récentes, Paris, Errance, Dossiers de protohistoire, 2, p. 121-135.

DÉCHELETTE J., 1914, Manuel d'archéologie préhistorique, celtique et gallo-romaine. II : Archéologie celtique ou protohistorique. 3 : Second âge du Fer, époque de La Tène, Paris, Picard.

DeHn R., 2005, « Das Oppidum Tarodunum bei Kirchzarten », in BR ÄUNING A. (dir.), Kelten an Hoch- und Oberrhein, Führer zu archäologischen Denkmälern in Baden-Württemberg, 24, Stuttgart, Theiss, p. 86-89.

FERNÁNDEZ-GÖTZ M., 2012, « Die Rolle der Heiligtümer bei der Konstruktion kollektiver Identitäten : Das Beispiel der treverischen Oppida », Archäologisches Korrespondenzblatt, 42, p. 509-524.

FERnÁNDEZ-Götz M., 2014a, « Central places and the construction of collective identities in the middle Rhine-Moselle region ", in Popa C. N., STOddart S. (dir.), Fingerprinting the Iron Age. Approaches to identity in the European Iron Age. Integrating South-Eastern Europe into the debate, Oxford, Oxbow, p. 175-186.

FERNÁNDEZ-GÖTZ M., 2014b, Identity and power. The transformation of Iron Age societies in Northeast Gaul, Amsterdam Archaeological Studies, 21, Amsterdam, Amsterdam University Press, $306 \mathrm{p}$.

FichtL S., 2005, «Murus et pomerium : réflexions sur la fonction des remparts protohistoriques », Revue archéologique du Centre de la France, t. 44, p. 55-72.

FICHTL S., 2010, «Les places publiques dans les oppida», L'Archéologue, $\mathrm{n}^{\circ}$ 108, p. 36-40.

FICHTL S., 2012, « Du "refuge" à la ville. 150 ans d'archéologie des oppida celtiques », in OLIVIER L. (dir.), Le Musée d'archéologie nationale et les Gaulois du XIX $X^{e}$ au XXI siècle, Antiquités Nationales, numéro spécial, Paris, Musée d'archéologie nationale, p. 81-98.

FISCHER F., 2001, « Oppidum Heidengraben », in Rieckhoff S., Biel J. (dir.), Die Kelten in Deutschland, Stuttgart, Theiss, p. 351-353.
Gruel K., Buchsenschutz O., 2015, « Un urbanisme perché », in BuchSENSChutz O. (dir.), L'Europe celtique à l'âge du Fer: VIII $-I^{e r}$ siècles, Paris, PUF, p. 307-315.

Kaenel G., Curdy P., Carrard F., 2004, Loppidum du Mont Vully: un bilan des recherches 1978-2003, Fribourg, Éditions Universitaires Fribourg, Archéologie fribourgeoise, 20, $280 \mathrm{p}$.

KřIVÁNEK R., DANIELISOVÁ A., DRDA P., 2013, Geofyzikalní prüzkum oppid v Čechách. Geophysical survey of oppida in Bohemia, Prague, Académie des Sciences de la République Tchèque, Archeologické studijní materiály, 23, 158 p.

KüSTER H., 1992, « Vegetationsgeschichtliche Untersuchungen », in MAIER F., GEILENBRÜGGE U., Hahn E., KöHLER H.-J., Sievers S., Ergebnisse der Ausgrabungen 1984-1987 in Manching, Stuttgart, Steiner, Die Ausgrabungen in Manching, 15, p. $433-476$.

Küster H., 2013, « Neue botanische Analysen an Fundgut aus dem latènezeitlichen Manching ", in Sievers S., Leicht M., Ziegaus B. (dir.), Ergebnisse der Ausgrabungen in Manching-Altenfeld 1996-1999, Reichert, Wiesbaden, p. 737-744.

Metzler J., 2006, « Religion et politique. L'oppidum trévire du Titelberg », in Goudineau C. (dir.), Religion et société en Gaule, Paris, Errance, p. 191-202

Metzler J., 2008, « Du Titelberg à Trèves. De l'oppidum gaulois à la ville romaine », in CASTELLA, F., MEYLAN Krause M.-F. (dir.), Topographie sacrée et rituels. Le cas d'Aventicum, capitale des Hélvètes, Actes du colloque international d'Avenches, 2-4 novembre 2006, 43, Bâle, Archéologie suisse, Antiqua, p. 155-165.

Metzler J., Méniel P., Gaeng C., 2006, « Oppida et eśpaces publics », in Haselgrove C. (dir.), Celtes et Gaulois, l'archéologie face à l'histoire. 4: Les mutations de la fin de l'âge du Fer, Actes de la table ronde de Cambridge, 7-8 juillet 2005, Glux-en-Glenne, Centre Archéologique Européen, Collection Bibracte, 12/4, p. 201-224.

MöldERS D., 2009, Die handwerkliche Produktion im Oppidum Bibracte (Mont Beuvray) im Spiegel der eisernen Werkzeuge und Werkabfälle der Ausgrabungen von Jacques-Gabriel Bulliot im 19 Jahrhundert, Glux-en-Glenne, Centre Archéologique Européen, Collection Bibracte, 18, 203 p.

Nickel C., Thoma M., Wigg-Wolf D., 2008, Martberg. Heiligtum und Oppidum der Treverer I. Der Kultbezirk. Die Grabungen 1994-2004, Koblenz, Gesellschaft für Archäologie an Mittelrhein und Mosel, 692 p.

Poux M., Demierre M., 2015, Le sanctuaire de Corent (Puy-de-Dôme, Auvergne). Vestiges et rituels, Paris, CNRS éditions, Gallia, $62^{\mathrm{e}}$ Supplément, $707 \mathrm{p}$

RALSTON I. B. M., 1992, Les enceintes fortifiées $d u$ Limousin. Les habitats protohistoriques de la France non méditerranéenne, Paris, Éditions de la Maison des sciences de l'Homme, Documents d'Archéologie Française, 36, $190 \mathrm{p}$.
Rind M., 1994, Ausgrabung « Kanal II » des Bayerischen Landesamtes für Denkmalpflege 1980, Buch am Erlbach, Leidorf, Archäologie am Main-DonauKanal, 3, 59 p.

Schwarz K., Tillmann H., Treibs W., 1965-1966, « Zur spätlatènezeitlichen und mittelalterlichen Eisengewinnung auf der südlichen Frankenalb bei Kelheim ", Jahresbericht der Bayerischen Bodendenkmalpflege, 6/7, p. 35-66.

Sievers S., 2010, « Zur Architektur der keltischen Oppida : zwischen Tradition und Innovation », in Trebsche P., Müller-SCheessel N., Reinhold S. (dir.), Der gebaute Raum. Bausteine einer modernen Architektursoziologie vormoderner Gesellschaften, Waxmann, Münster, Tübinger Archäologische Taschenbücher, 11, p. 307-324.

Sмiтн M. L., 2008, « Urban empty spaces. Contentious places for consensus-building », Archaeological Dialogues, 15, p. 216-231.

Stegmaier G., 2014, «'Die Stadt im Kornfeld'. Untersuchungen zur Wirtschafts- und Siedlungsstruktur des Oppidums Heidengraben », in Hornung S. (dir.), Produktion - Distribution - Ökonomie. Siedlungs- und Wirtschaftsmuster der Latènezeit, Akten des internationalen Kolloquiums in Otzenhausen, 28.-30. Oktober 2011, Bonn, Habelt, Universitätsforschungen zur prähistorischen Archäologie, 258, p. 271-292.

Tuschwitz A., 2015, Das Oppidum von Kelheim, Marburg, Beltz, Oppida Celtica, 2, 102 p.

UeRPMANn M., Uerpmann H.-P., 2006, «Tierknochenfunde aus dem Oppidum Heidengraben bei Grabenstetten (Kr. Reutlingen) », in Knopf T., Der « Heidengraben » bei Grabenstetten. Archäologische Untersuchungen zur Besiedlungsgeschichte, Bonn, Habelt, Universitätsforschungen zur prähistorischen Archäologie, 141, p. 263-29o.

WENDLING H., 2005, « Ausgrabungen in der mittelund spätlatènezeitlichen Großsiedlung von Kirchzarten-Zarten 'Rotacker', Kreis BreisgauHochschwarzwald », Archäologische Ausgrabungen in Baden-Württemberg, 2004, p. 107-110.

Wendling H., 2006, « Töpfer, Schmiede, Münzmeister. Nachweise mittel- und spätlatènezeitlichen Handwerks in Tarodunum, Gde. Kirchzarten, Kreis Breisgau-Hochschwarzwald », Archäologische Ausgrabungen in Baden-Württemberg, 2005, p. 107-110.

WINGER K., 2015, Baubefunde und Siedlungsentwicklung der Südumgehung im Oppidum von Manching, Wiesbaden, Reichert, Die Ausgrabungen in Manching, 20, $294 \mathrm{p}$.

Zeeb-Lanz A., 2016, « Münzen - Mauern - Zangentore. Zehn Jahre Forschungen auf dem keltischen Donnersberg in der Nordpfalz (2004-2014)", in Косн M. (dir.), Archäologentage Otzenhausen 2. Archäologie einer Großregion. Internationales Symposium zur Archäologie in der Großregion in der Europäischen Akademie Otzenhausen 19.-22. Februar 2015, Europäische Akademie Otzenhausen, Nonnweiler, p. 21-55. 